\title{
Les modalités iconiques dans le discours médié par ordinateur : du neuf dans l'interaction?
}

\section{Célia Schneebeli}

\section{(2) OpenEdition}

\section{Journals}

Édition électronique

URL : http://journals.openedition.org/esa/3126

DOI : $10.4000 /$ esa. 3126

ISSN : 2650-2623

\section{Éditeur}

Société de stylistique anglaise

\section{Édition imprimée}

Date de publication : 31 décembre 2018

Pagination : 45-66

ISBN : 978-2-36442-083-0

ISSN : 2116-1747

Référence électronique

Célia Schneebeli, «Les modalités iconiques dans le discours médié par ordinateur : du neuf dans l'interaction? », Études de stylistique anglaise [En ligne], 13 | 2018, mis en ligne le 20 mars 2019, consulté le 20 mars 2020. URL : http://journals.openedition.org/esa/3126 ; DOI : https://doi.org/ 10.4000/esa.3126 


\title{
Les modalités iconiques dans le discours médié par ordinateur : du neuf dans l'interaction?
}

\author{
Célia SCHNEEBELI \\ Université de Bourgogne \\ EA 2610 - Centre Interlangues
}

\section{Introduction}

Dans ce qui est sans doute le premier ouvrage de référence consacré à la Communication Médiée par Ordinateur (CMO), Susan C. Herring présentait l'utilisation des émoticônes et de ce qu'elle nommait alors " other graphics " comme un des traits propres à l'interaction digitale (" the computer-mediated register has unique features of its own, such as the use of 'emoticons' [...] and other graphics ", Herring 1996, 3). D'autres linguistes ont depuis fortement nuancé cette idée d'exclusivité, comme Markus Bieswanger qui note que l'utilisation d'images dans l'interaction est loin d'être spécifique à la CMO (Biewanger 2013, 470). Il suffit d'ailleurs pour s'en convaincre de lire le chapitre sur l'écriture dans le Nouveau Dictionnaire Encyclopédique des Sciences du Langage (Ducrot \& Schaeffer 1995, 301-310), où il est question des systèmes pictographiques que l'on trouve dans la plupart des civilisations. Inversement, on peut aussi objecter que l'interaction médiée par ordinateur était à l'origine exclusivement verbale et textuelle en raison des possibilités techniques d'affichage initialement bien plus limitées qu'aujourd'hui. Il demeure néanmoins que l'utilisation fréquente d'images de toutes sortes comme moyen d'expression est désormais perçue comme une des caractéristiques saillantes du discours médié par ordinateur, particulièrement dans l'interaction entre usagers, que ce soit dans les courriers électroniques, sur les réseaux sociaux, ou encore via les messageries instantanées. Depuis le premier ouvrage de Herring, d'autres 
LES MODALITÉS ICONIQUES DANS LE DISCOURS MÉDIÉ

PAR ORDINATEUR : DU NEUF DANS L'INTERACTION ?

déclinaisons des modalités iconiques se sont par ailleurs développées, particulièrement les images animées, comme les gifs et les vidéos, qui sont venues s'ajouter plus récemment aux images fixes dans les interactions sur certaines plateformes comme Facebook, Twitter, ou Tumblr.

En réalité, la question n'est pas tant de savoir si ces traits sont propres ou non à la CMO mais de déterminer ce que le recours aux modalités (au sens large de moyens d'expression) iconiques apporte à l'interaction médiée par ordinateur, ainsi que son statut dans l'interaction. Dans quelle mesure ces modalités, qui sont d'ordinaire rattachées au nonverbal, interagissent-elles voire se substituent-elles aux modalités verbales (c'est-à-dire, à l'écrit, le matériel lexical et morpho-syntaxique)? Quelle est leur place dans l'interaction? Voilà les questions auxquelles cet article va s'attacher à répondre en examinant un corpus d'interactions prélevé dans des commentaires postés sur le réseau social Facebook.

\section{Corpus}

Facebook est un média idéal pour étudier les modalités iconiques puisqu'à la différence d'autres plateformes, comme YouTube ou Instagram, il permet à l'utilisateur de se servir de toutes les formes d'image que l'on peut trouver dans les interactions sur Internet : émoticônes, émojis, autocollants, gifs, images et vidéos. Les pages publiques offrent en outre au linguiste une ressource inépuisable d'interactions publiques, qui ne posent pas de problèmes de confidentialité. Cependant, en parcourant ces pages, on se rend assez rapidement compte qu'il est difficile, voire impossible, de trouver un corpus totalement représentatif de la manière dont les utilisateurs se servent des modalités iconiques à leur disposition. En effet, l'utilisation de ces ressources dépend étroitement du profil des utilisateurs de la page, notamment de leur classe d'âge, de leurs domaines d'intérêt, et de leur degré de compétence en matière d'outils numériques, ainsi que du sujet et du genre de la page. Il est évident que l'on ne trouve pas les mêmes types d'échanges, tant du point de vue du fond que de la forme, sur la page d'un groupe d'universitaires (où on trouve peu de modalités iconiques, sans doute parce que ces dernières sont considérées comme faisant partie du registre familier ou informel et sont donc encore peu utilisées sur les pages liées aux activités professionnelles), d'un site humoristique dédié au partage d'images et de vidéos (où on trouve logiquement une grande quantité de gifs et d'images modifiées dans les 
commentaires) ou d'un club d'amateurs de chats de race (où le type d'image le plus fréquemment rencontré dans les messages est la photo personnelle utilisée avec un but illustratif). L'étude porte ici sur la page publique d'une équipe de hockey sur glace nord-américaine, les Flyers de Philadelphie. Les pages officielles des équipes sportives ont l'avantage d'être fréquentées par un public assez large et varié. Les interactions sont également particulièrement nombreuses sur ces pages, qui sont très régulièrement alimentées. Cela était encore plus vrai au moment où la collecte de l'échantillon a été faite, en pleine période des séries éliminatoires de la ligue d'Amérique du nord (LNH), pour laquelle l'équipe de Philadelphie s'était qualifiée. Il s'agit d'une époque de l'année où les passionnés suivent les pages des équipes de près, que ce soit pour encourager leur équipe, la critiquer ou provoquer l'adversaire. La page choisie présente en outre des occurrences d'utilisation de toutes les modalités iconiques, utilisées dans des fonctions diverses, ce qui rend son choix d'autant plus pertinent. Au final, l'échantillon étudié comporte l'intégralité des commentaires écrits en réaction à 4 messages postés par les administrateurs de la page des Flyers de Philadelphie entre le 19 et le 22 avril 2018, ce qui totalise 1157 commentaires. Sur ces 1157 commentaires, qui mêlent réactions au message initial et commentaires sur les commentaires, 213 messages utilisent des images, soit $18,4 \%$. On peut donc d'ores et déjà constater que le recours à l'image n'est pas aussi fréquent qu'on pourrait le penser et que les modalités verbales sont encore largement préférées par les utilisateurs. On reviendra plus loin sur ce point, qui a déjà été noté dans d'autres études (par exemple Herring 2012, Schneebeli 2018).

Il faut enfin clore cette présentation du corpus étudié par une précision d'ordre terminologique. Peu d'articles portent sur l'utilisation des images dans l'interaction médiée par ordinateur. Les articles anglophones parlent le plus fréquemment de modalités "graphiques", là où les chercheurs francophones utilisent indifféremment les termes de modalités "graphiques" ou "iconiques". Dans la mesure où le terme "graphique " est très large, au point d'inclure normalement l'écriture, et en considérant qu'on s'intéresse ici exclusivement à l'utilisation d'images, il semble plus pertinent d'utiliser le terme "iconique", au sens de relatif à l'image. Il s'agit du choix fait dans cet article. 
LES MODALITÉS ICONIQUES DANS LE DISCOURS MÉDIÉ

PAR ORDINATEUR : DU NEUF DANS L'INTERACTION ?

\section{Le discours médié par ordinateur : un nouveau type de discours?}

Les commentaires écrits sur Facebook appartiennent à ce que l'on appelle le discours médié par ordinateur, DMO en français et CMD en anglais. Voici la définition qu'en donnent Susan C. Herring et Jannis Androutsopoulos $(2015,128)$ :

Computer-mediated discourse (CMD) is the communication produced when human beings interact with one another by transmitting messages via networked or mobile computers, where " computers " are defined broadly to include any digital communication device.

Facebook fait plus exactement partie de ce qui est souvent nommé, peut-être un peu abusivement, le web 2.0, c'est à dire une génération de sites et plateformes Internet davantage tournés vers l'interaction, qui a commencé à se développer au milieu des années 2000. A ses débuts, Facebook ne permettait pas vraiment d'interagir. Quand le site a été lancé en 2004 à Harvard, il s'agissait en quelques sortes d'un trombinoscope étudiant adapté à Internet. L'utilisateur pouvait alors seulement remplir son profil, consulter les profils des autres utilisateurs, se connecter à ses amis et former des groupes liés à ses centres d'intérêt. Des fonctionnalités supplémentaires ont été ajoutées au fur et à mesure, comme la possibilité de s'envoyer des messages (2005), de publier des photos (2005), des jeux (2007), la publication d'un statut, qui permet de partager son humeur, ses pensées ou son activité (2008) ou encore le fameux bouton "like" et son pouce levé (2009), complété par les autres boutons de réaction (2016). Si bien que le site, partant d'un format existant, celui du trombinoscope ou de l'annuaire étudiant, a fini par former quelque chose de nouveau, sans véritable précédent. Qu'en est-il, alors, de son discours ? Est-il aussi novateur que son support ? Pour répondre à cette question, on peut commencer par noter que Facebook, dans sa configuration actuelle, fait partie de ce que Susan C. Herring appelle "interactive multimodal platforms (IMPs)": "Web 2.0 platforms that support a convergence of channels or 'modes' (text, audio, video, images) for user-to-user communication" (Herring 2016, 398). Autrement dit, Facebook fait partie de ces sites et plateformes Internet qui permettent aux usagers d'utiliser plusieurs modes de communication : le texte, le son, la vidéo, mais aussi l'image statique. Sur Facebook, le discours est donc largement multimodal. Cette utilisation d'autres modes que le texte (au sens précis 
de suite de signes linguistiques qui forme un écrit) et le recours à d'autres systèmes sémiotiques que le langage verbal dans la conversation sur Internet est considérée par Herring comme un phénomène émergent (" [a]nother emergent Discourse 2.0 phenomenon is the use of channels other than text, and semiotic systems other than verbal language, to carry on "conversational" exchanges ", Herring 2013, 16). Il ne faut cependant pas exagérer le degré de nouveauté des phénomènes discursifs engendrés par les moyens qu'offre le site. Comme le souligne également Herring, contrairement à ce que le terme de "nouveaux médias " sous-entend, une bonne partie de ce qu'on trouve dans le web 2.0 en matière de discours n'a rien de nouveau, ne serait-ce que parce que le mode par excellence de l'interaction médiée par ordinateur reste le texte (" text remains the predominant channel of communication among web users, whether it be in blogs, microblogs, wikis, comments on news sites, or web discussion forums ", Herring 2013, 9). Le discours du web 2.0 peut donc difficilement être qualifié de révolutionnaire. Par ailleurs, contrairement à ce qu'affirmaient certains chercheurs appartenant à ce qu'Androutsopoulos (2006, 42) appelle "'first wave' of linguistic CMC studies ", c'est-à-dire les premiers chercheurs à avoir travaillé sur la communication médiée par ordinateur, il n'y a pas de langage spécifique à la CMO (voir Bieswanger 2013, 465 pour une description détaillée des études en question). Ce qui a été appelé 'Netspeak' par le linguiste David Crystal, terme qui a connu un certain succès populaire, n'existe tout simplement pas, et de nombreuses études plus récentes le prouvent (on peut citer par exemple Herring 2007 et 2012, ou encore Bieswanger \& Intermann 2011). Le discours médié par ordinateur utilise les mêmes modalités que le discours produit dans d'autres environnements. Il a néanmoins des traits caractéristiques, dont l'utilisation récurrente d'images dans différentes fonctions. Celle-ci n'est cependant pas exclusive à l'interaction en ligne, comme cela a été dit en introduction.

\section{Les modalités iconiques disponibles sur Facebook}

Herring et Dainas dénombrent 6 formes de modalités iconiques pouvant être utilisées dans l'interaction en ligne (Herring \& Dainas 2017) :

- Les émoticônes, qui sont des représentations d'expressions faciales utilisant les caractères disponibles sur un clavier d'ordinateur, comme on en trouve dans le commentaire suivant : 


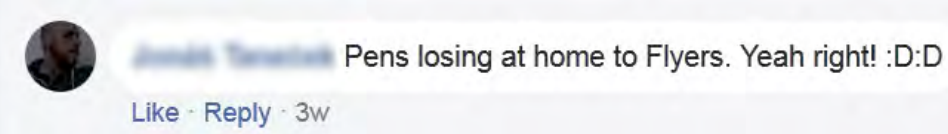

Il n'y a presque pas d'émoticônes dans l'échantillon car l'interface de Facebook les transforme automatiquement en émoji (petite icône stylisée, voir ci-dessous) de sens équivalent. Les seuls émoticônes qui apparaissent sont ceux qui sont mal formés, et donc non détectés par l'interface (c'est le cas dans l'exemple ci-dessus, où il manque un espace entre les deux émoticônes), ou ceux pour lesquels Facebook ne propose pas d'équivalent en émoji (par exemple ${ }^{\wedge}$, qui représente un visage qui sourit avec les yeux).

- Les émojis, qui sont des petites icônes digitales, de même taille que les caractères du clavier, et qui peuvent représenter toutes sortes de choses, des visages aux objets en passant par les animaux ou de nombreux pictogrammes. Le message suivant en utilise deux :

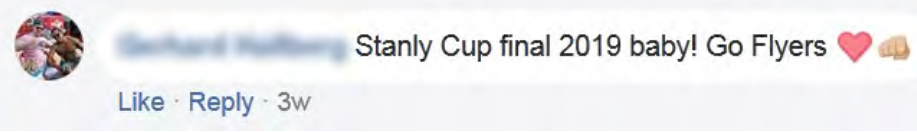

- Les autocollants, qui sont des petites images de formes diverses comparables aux émojis mais de taille supérieure et pouvant intégrer du texte. Elles peuvent elles aussi représenter toutes sortes de choses mais se concentrent la plupart du temps sur les expressions faciales et les personnages :

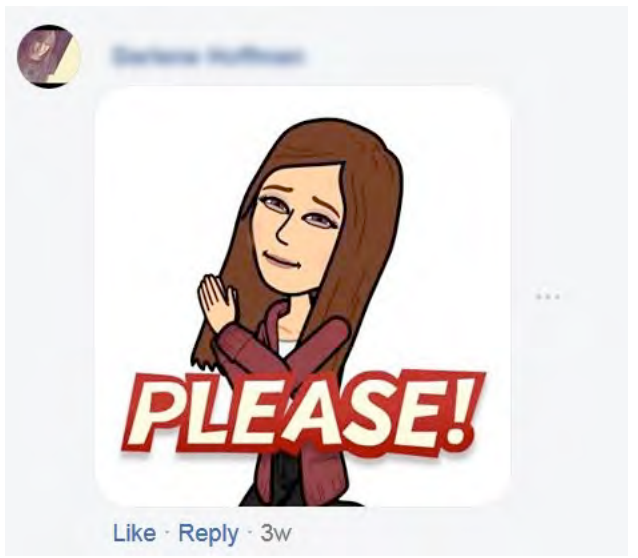


- Les gif, nom qui désigne à l'origine un format d'image (Graphics Interchange Format). Sur les réseaux sociaux le terme gif désigne plutôt une image animée composée d'une séquence de plusieurs images enchaînées diffusée en boucle, souvent extraite de films, de séries ou de dessins animés. L'utilisation de gif n'est possible sur Facebook que depuis mi-2017, comme dans le commentaire suivant, où le personnage principal du film Ted, un ours en peluche, envoie un baiser avec sa main :

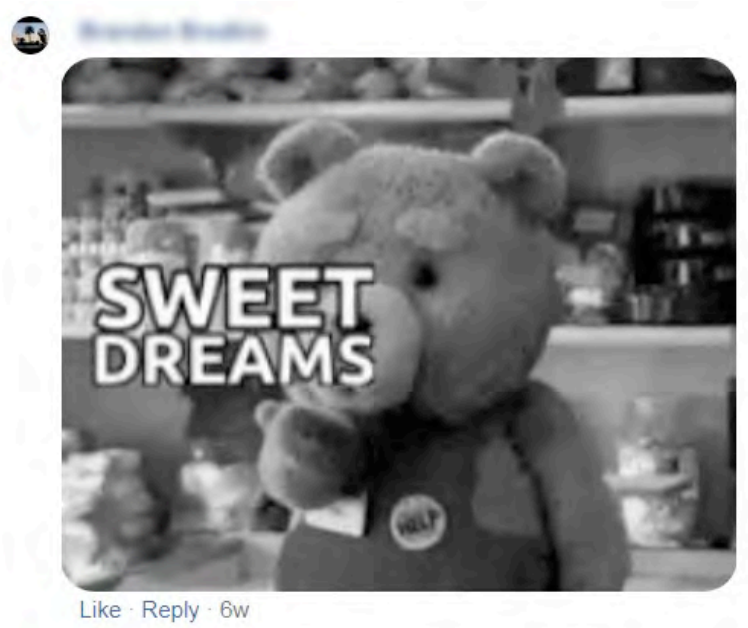

- Les vidéos, qui sont directement insérées dans le commentaire et apparaissent sous forme de miniature avec un titre, et sur laquelle il suffit de cliquer pour jouer la vidéo :

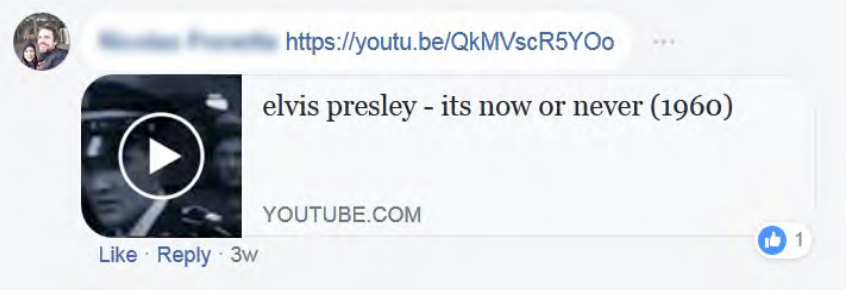

- Les images, qui forment la catégorie la plus large et la mieux représentée. Il peut en fait s'agir d'images fixes, de photos ou dessins, qui apparaissent sous forme de fichier rectangulaire ou carré : 


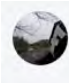

Hakstol in the dressing room rn like

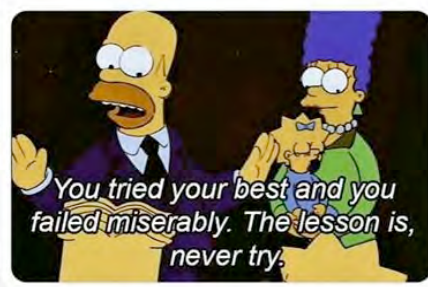

Like - Reply - 3w

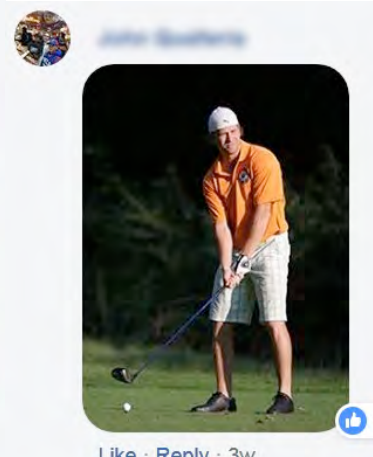

Like · Reply · 3w

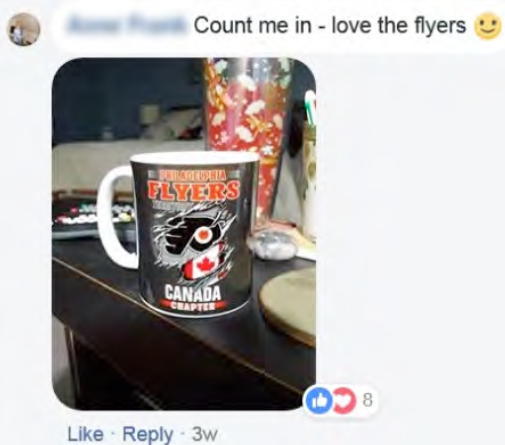

Toutes ces modalités sont disponibles sur Facebook, et la plupart sont même directement proposées à l'utilisateur via un bouton spécifique dans les options des commentaires :

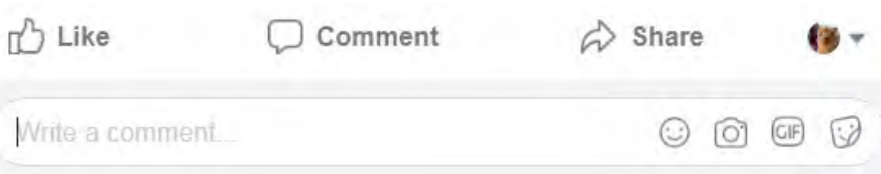

En bas à droite dans la zone réservée au texte du commentaire se trouvent quatre boutons permettant d'insérer directement une image. Le premier permet d'accéder directement au répertoire d'émojis, le deuxième permet d'insérer une photo ou une image, le troisième donne accès au répertoire de gifs, et le dernier ouvre le répertoire d'autocollants. Quant aux vidéos, elles s'affichent en miniature une fois le lien copié-collé dans le commentaire.

Comment ces modalités iconiques s'articulent-elles avec le matériau verbal dans les messages? Pour répondre à cette question, il faut examiner 
la manière dont elles intègrent (ou non) le texte et quel statut elles occupent dans le message.

\section{Les modalités iconiques intégrées au texte du message}

Comme on l'a dit plus haut, l'interaction en ligne reste essentiellement textuelle, c'est-à-dire qu'elle a pour mode privilégié le texte, plutôt que les échanges vidéo ou audio. Certaines images, plus exactement les émoticônes et les émojis, sont complètement intégrées au texte car elles sont incorporées au niveau même de l'énoncé. Cela semble assez logique concernant les émoticônes puisqu'ils sont formés avec les mêmes caractères que le code linguistique à proprement parler. Les émojis, eux, sont bien des images mais ils sont conçus pour être intégrés directement au sein des phrases grâce à leur format à peu près aligné sur celui des caractères. Emoticônes et émojis apparaissent donc dans le linéaire de l'énoncé et leur valeur est partie prenante de l'organisation séquentielle du discours. Ils sont d'ailleurs rarement utilisés seuls dans le corpus (cela n'arrive que dans trois messages), même si c'est tout à fait possible.

La plupart du temps, ils apparaissent en fin d'énoncé. Leur fonction est alors souvent d'appuyer une réaction émotionnelle déjà explicitée par le texte. Par exemple, dans le message suivant, l'émoji exprimant la tristesse confirme le contenu propositionnel du message, "I'm sadly disappointed " :

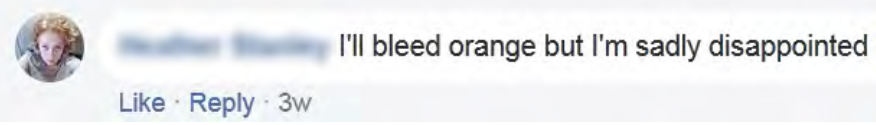

L'expression faciale représentée par l'émoji est redondante avec le contenu du message et l'émotion exprimée par ce dernier. Cette première fonction va de pair avec une autre fonction proche, examinée en détail par Eli Dresner et Susan C. Herring, qui est celle de marqueur de force illocutoire (Dresner \& Herring 2010). Autrement dit, ils servent à indiquer l'attitude du locuteur par rapport à son énoncé et comment le message doit être interprété. C'est le cas dans les trois messages ci-dessous : 


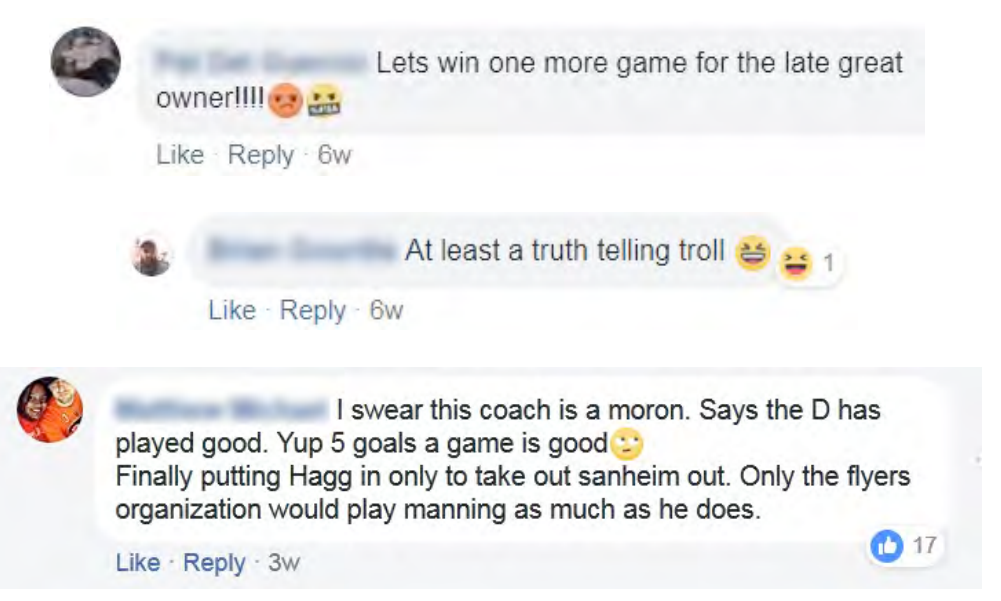

Dans le premier message, les deux émojis précisent que le commentateur est irrité, ce que le message en lui-même ne dit pas. Dans le deuxième, le visage riant indique que le message n'est pas à prendre sérieusement. Enfin, dans le troisième, le visage qui lève les yeux au ciel dénote que le message, dont le contenu est plutôt mélioratif, n'est non plus à prendre littéralement et qu'il est en réalité ironique. Parfois, c'est l'émoji qui est en lui-même ironique, comme c'est le cas ici :

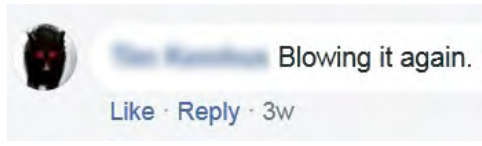

Le geste représenté par l'émoji n'est pas redondant avec le contenu $\mathrm{du}$ message. Les applaudissements viennent contredire le caractère dépréciatif du message et expriment indirectement l'agacement de l'utilisateur.

Les émojis constituent dans tous ces cas un moyen de compenser, dans le discours médié par ordinateur, l'absence d'indications données par l'intonation, les expressions faciales, les rires, les soupirs, et autres modalités kinésiques ou corporo-visuelles (Kerbrat-Orecchioni 1998, 137). Ces dernières sont une partie essentielle de l'interaction face-à-face comme l'ont montrées les études gestuelles modernes (par exemple Porcher et Calbris 1989, McNeill 1992, Kendon 2004, Calbris 2011). Les gestes, mouvements du corps et expressions faciales accompagnent, renforcent, précisent, nuancent, voire remplacent le discours proprement verbal. Les émojis semblent remplir des fonctions similaires dans l'interaction écrite 
en ligne. En raison de la nature séquentielle du discours médié par ordinateur, ils sont alors intégrés au texte à la manière d'une ponctuation et apparaissent systématiquement en fin d'énoncé.

Les émojis apparaissent aussi parfois en position médiane au sein de l'énoncé, avec un rôle de substitut lexical :

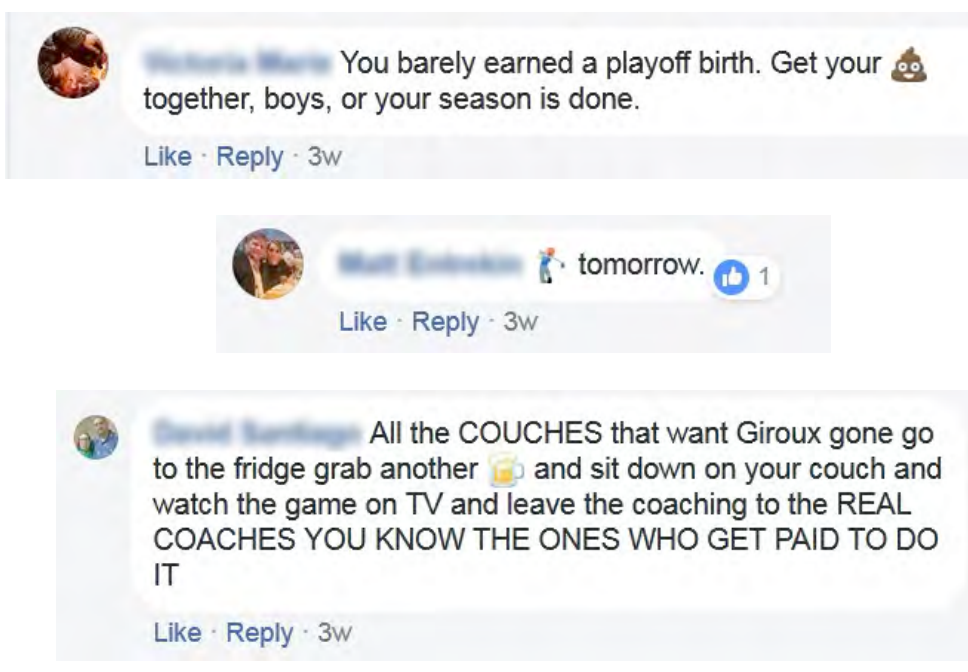

Dans le premier message, l'émoji remplace le nom "shit", dans le deuxième, l'émoji remplace le nom "golf" ou "golfing" (qui décrit l'activité que les joueurs vont pouvoir commencer dès le lendemain une fois éliminés), et dans le dernier message, c'est clairement le nom "beer" qui est remplacé par l'émoji qui représente une chope de bière. Dans ces exemples, les émojis sont donc utilisés en lieu et place des mots équivalents, qui peuvent être des noms, des verbes ou des adjectifs. Les émojis peuvent représenter des objets, des personnes ou animaux, mais aussi encore des gestes et des expressions faciales. Dans ce dernier cas, le geste peut avoir valeur de "pantomime " comme l'appelle McNeill (1992, qui développe le continuum de Kendon 1988), c'est-à-dire que le geste mime ce à quoi il fait référence. C'est le cas ici avec l'émoji représentant un golfeur, où l'activité à laquelle il est fait référence est mimée. Il peut aussi avoir une valeur d'"emblème", auquel cas sa signification symbolique est conventionnelle. Il n'y a pas d'exemple de ce genre dans le corpus mais on peut penser au cas où un commentateur utilise un émoji de pouce levé à la place du mot "ok" ou "great». Dans tous les cas, les 
émojis font partie de l'axe paradigmatique, c'est-à-dire de l'ensemble des choix possibles à cet endroit de l'énoncé au même titre que les mots. Il s'agit là d'une utilisation attendue des émojis puisqu'ils ont la plupart du temps une fonction référentielle, dans la mesure où il s'agit d'icônes qui représentent un objet, un être, une activité, au même titre qu'un mot. Cela n'est d'ailleurs pas propre aux émojis puisque c'est là le fondement même du rébus. Mais la fréquence d'utilisation des émojis rend cette pratique plus courante et contribue à montrer que la limite entre les modalités iconiques et les modalités verbales n'est pas toujours si nette que cela. D'ailleurs, en 2015, c'est un émoji appelé " face with tears of joy " qui a été choisi comme mot de l'année par l'équipe de l'Oxford English Dictionary. Cela montre combien ils tendent à être désormais considérés comme des marqueurs au moins textuels sinon verbaux.

\section{Les modalités iconiques non intégrées au texte du message}

Les autres modalités iconiques n'ont pas le même statut par rapport au texte et même par rapport au matériau proprement verbal de l'interaction. En premier lieu, elles ne sont pas du tout conçues techniquement pour intégrer une phrase. Elles sont toujours séparées du texte et insérées soit en-dessous, la plupart du temps, soit au-dessus. Elles sont en revanche toutes conçues pour être couplées avec du texte au sein d'un message. Par exemple, il est tout à fait possible, dans un message, d'écrire une phrase puis d'insérer un gif, une vidéo, un autocollant ou une image. L’image apparaîtra simplement séparée et non insérée dans le texte :

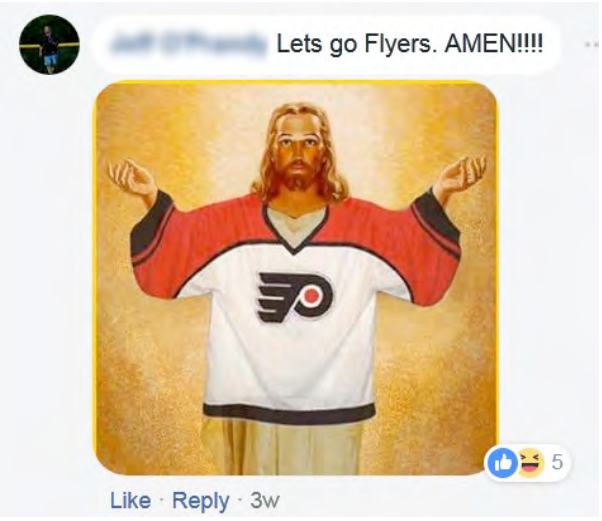


Dans l'esprit des utilisateurs, ces modalités semblent toutefois moins liées à l'emploi de texte que les émojis ou les émoticônes. Le cas du gif est le plus frappant car 64 des 65 messages utilisant un gif dans le corpus utilisent uniquement cette image sans écrire de texte. C'est aussi le cas du seul message utilisant un autocollant dans le corpus, ainsi que des trois messages utilisant une vidéo. Ils ne sont jamais précédés ou suivis de texte ajouté par l'utilisateur. Le recours aux images est, lui, plus contrasté puisque 41 messages sur 66 consistent uniquement en une image, ce qui veut dire que 25 messages utilisent à la fois du texte et une image.

Une question que l'on peut se poser est pourquoi ces modalités, particulièrement les gifs, sont si souvent utilisées seules. La réponse est loin d'être simple et claire. Une réponse possible est dans la richesse de ces modalités iconiques, particulièrement les gifs, qui sont des petites séquences d'images animées, par rapport aux émoticônes et aux émojis, qui consistent en des images fixes relativement simples voire stylisées puisqu'elles ne représentent de manière très simplifiée qu'une chose à la fois. C'est le postulat central de Tolins et Samermit (2016) : les gifs permettent de reproduire la part non-verbale qui revient aux gestes et expressions faciales dans la communication face à face. Pour cela, l'utilisateur sélectionne un gif montrant une personne ou un personnage faisant le geste ou l'expression souhaitée : lever les yeux au ciel, soupirer, rire à gorge déployée, se prendre la tête dans les mains, ou encore avoir l'air désespéré. Et en effet, dans le corpus, plus de la moitié des gifs utilisés (34 sur 65) représentent une personne faisant une action de ce type qui peut être interprétée comme une réaction à ce qui est dit par le message premier ou un autre commentaire. Pour Tolins et Samermit, le gif permet de représenter exactement et directement cette réaction, contrairement aux émoticônes et émojis, qui ont un fonctionnement plus symbolique ou iconique (" reproducing these embodied actions functionally distinguishes GIFs from other embodied displays in text messaging such as emoticons, which are symbolic and abstract in nature rather than quotative ", Tolins $\&$ Samermit 2016, 77). Cette première réponse n'est que partielle car il faut ajouter à cela que si les gifs apparaissent le plus souvent sans accompagner de texte, ils comportent en revanche souvent du texte à l'intérieur de l'image. Sur les 65 gifs utilisés dans le corpus, 26 comportent en effet du texte incrusté dans l'image. Or ce texte a le plus souvent une fonction de réplique accompagnant le geste ou l'expression faciale. Avec le gif, on obtient dans ce cas l'équivalent d'une réplique qui comporte une partie 
verbale (la réplique incrustée dans l'image) et offre les modalités kinésiques qui vont avec (regard, expression faciale, gestuelle). Cela explique sans doute qu'il constitue le plus souvent à lui seul une réplique. Bien sûr le gif ne sert pas qu'à mimer la réaction qu'aurait eue le locuteur dans un échange en face-à-face. Il sert aussi par exemple à se moquer de l'interlocuteur en lui renvoyant une image déplaisante de lui-même. C'est ce que fait un utilisateur avec le gif suivant, qui représente une petite fille en pleine colère avec l'énoncé "not jealous at all", et qui est utilisé en réponse à un supporter de l'équipe adverse qui critique un joueur de premier plan :

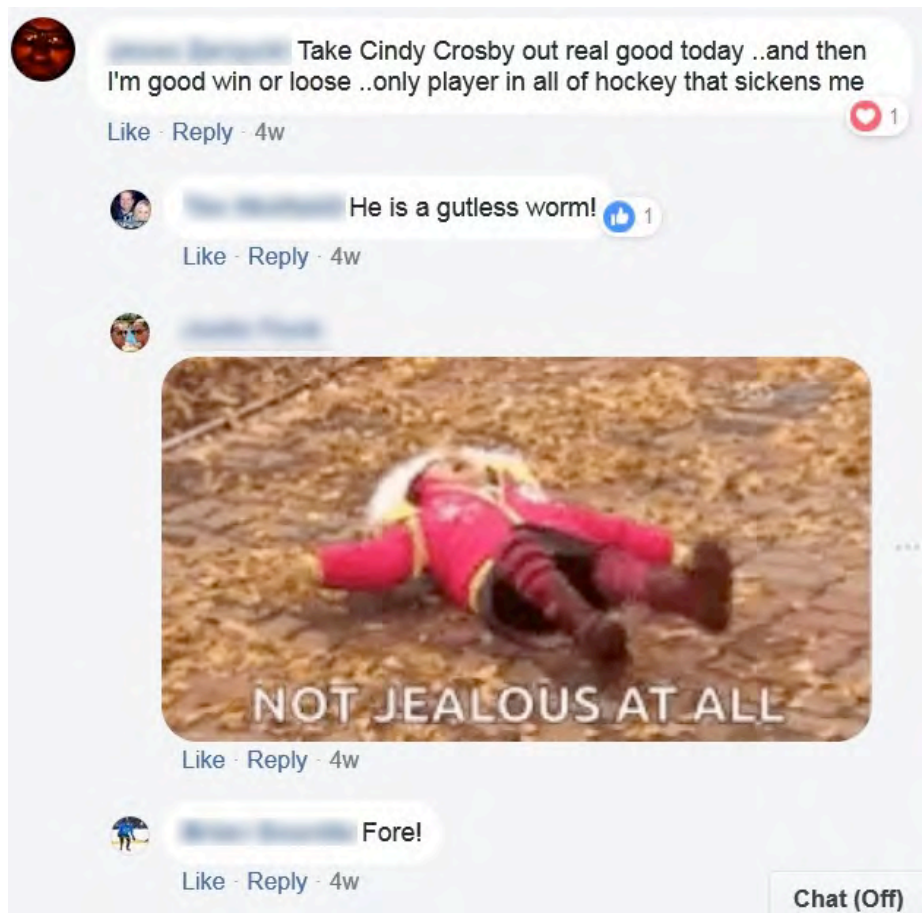

Le gif peut aussi servir à provoquer les supporters de l'équipe adverse ou à encourager l'équipe soutenue, par exemple en montrant de courtes vidéos de matches ou de joueurs sous un angle (dé)favorable. Pragmatiquement, le choix de l'image est enfin aussi un moyen de se présenter, voire se positionner socialement grâce aux références culturelles que comporte l'image (voir Miltner \& Highfield 2017 à ce sujet), ou tout simplement de faire de l'humour. 
Tout ceci est aussi valable pour les images, même si elles sont plus souvent employées pour accompagner du texte et qu'elles sont beaucoup moins fréquemment employées par l'utilisateur pour mimer sa réaction à un message. Quand elles accompagnent un texte, elles sont régulièrement utilisées avec un but purement illustratif (montrer la chose dont on parle), ce qui est le cas dans les deux messages suivants :
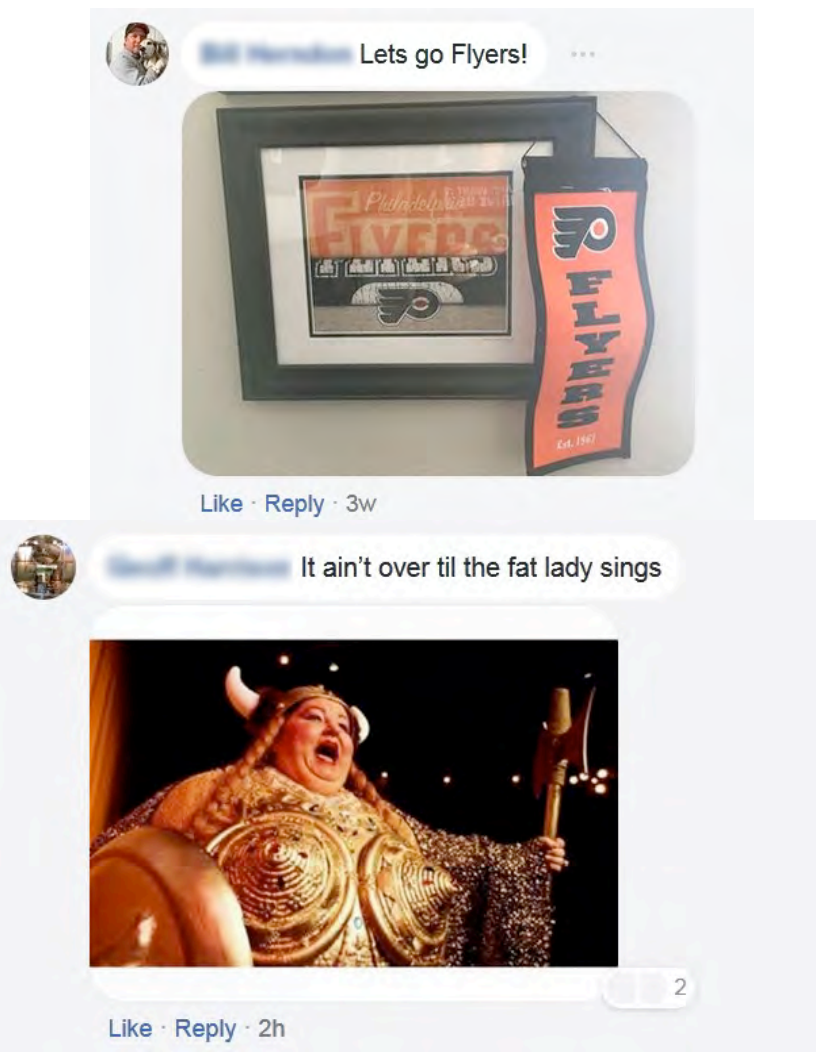

Qu'elles accompagnent ou non du texte, les images servent elles aussi à provoquer les supporters de l'équipe adverse, tout comme encourager son équipe. Enfin, comme les gifs, elles contiennent régulièrement du texte puisque 29 des 66 images utilisées dans le corpus comportent du texte incrusté à l'intérieur de l'image, à l'exemple des deux messages ci-dessous : 

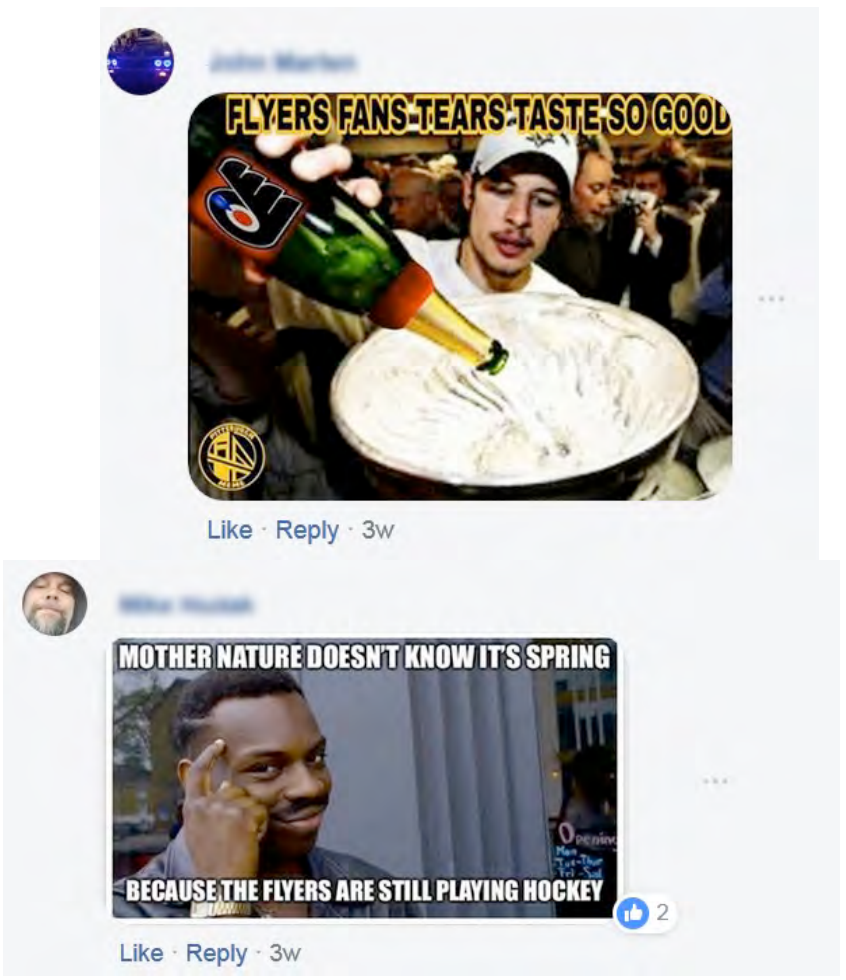

Cela laisse au final une proportion moins importante de messages qui utilisent uniquement de l'image sans aucune forme de texte (environnant ou incrusté), à l'exception des gifs, qui sont tout de même 39 à apparaître sans accompagner de texte et sans aucun texte incrusté. Ces constatations semblent en tout cas confirmer les dires de Herring et Androutsopoulos: "Perhaps surprisingly, studies of multimodal CMD show that text remains popular, even as communication in other modes increases. [...] At the same time, text-only CMD is on the decline" (Androutsopoulos et Herring 2015, 143).

\section{Place des modalités iconiques dans la structure de l'interaction}

Penchons-nous pour finir sur la place des images dans la structure non plus du discours que constitue le message, mais de l'interaction ellemême. Les chercheurs en communication médiée par ordinateur 
appliquent depuis déjà un certain temps les outils de l'analyse interactionnelle aux échanges virtuels, particulièrement l'analyse liée à la gestion des tours de parole. Le principe d'" alternance des tours" s'applique en effet à la CMO comme à un grand nombre de systèmes communicatifs. Sur Facebook, l'interaction est de deux types: les commentaires qui répondent au message initial posté sur une page, et les commentaires qui répondent à d'autres commentaires. Dans tous les cas, on considère qu'il y a interaction dès lors qu'il y a réponse à un message initial, que ce soit le message posté sur la page ou un autre commentaire. Dans les deux cas, chaque utilisateur poste un message à son tour, comme dans l'interaction orale. Il s'agit cependant ici d'interaction asynchrone puisque les utilisateurs ne sont pas nécessairement présents en même temps. La communication est également unidirectionnelle, c'est-à-dire que les utilisateurs ne peuvent interagir pendant la production du message. Contrairement à la communication orale, les tours ne se superposent donc jamais. Enfin, il faut aussi ajouter un élément à la liste que fait Véronique Traverso des unités pouvant constituer un tour de parole dans l'interaction face à face. Il peut s'agir d'" un mot [...], une phrase simple [...] ou complexe, ou encore un syntagme [...]" (Traverso 1999, 31), mais aussi, dans la CMO, d'une image. En effet, comme on l'a vu plus haut, toutes les modalités iconiques peuvent constituer un tour de parole dans la conversation en ligne. C'est le cas, dans le corpus, de 111 messages, soit 9,5\% des 1157 messages, qui sont constitués d'une image seule et constituent donc un tour de parole. L'image est alors traitée comme formant une réplique à elle seule, à laquelle le tour adjacent peut d'ailleurs répondre par une autre image seule, comme c'est le cas dans cet échange : 


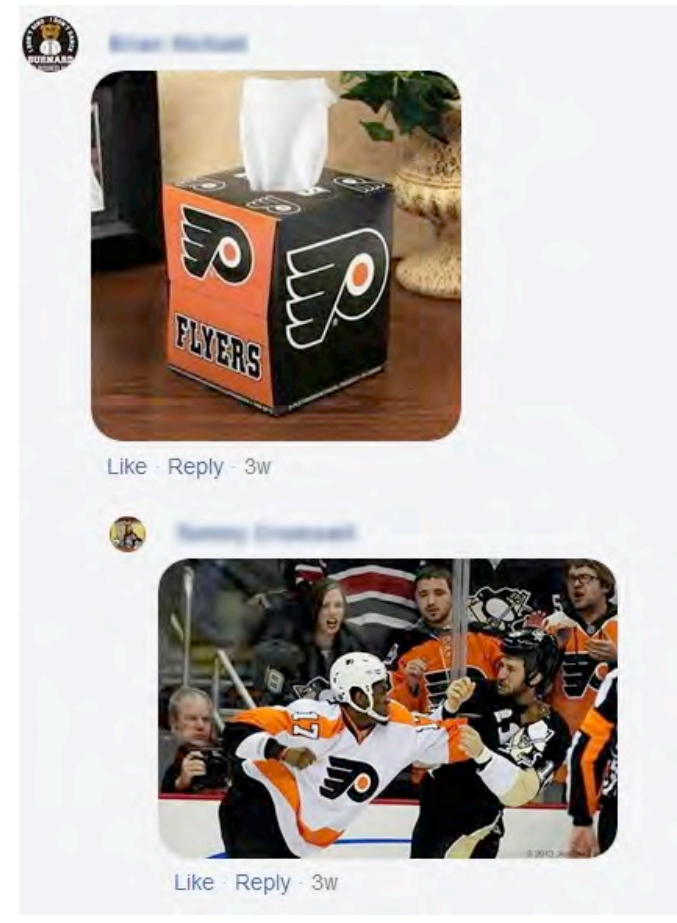

Le deuxième utilisateur répond au premier (qui le provoquait en lui montrant une boîte de mouchoirs portant le logo de son équipe, les Flyers de Philadelphie) au moyen d'une image qui constitue une forme de contreattaque (l'image montre un joueur de l'équipe de Philadelphie frappant un joueur de l'équipe adverse, celle de Pittsburgh). Face à ce type d'échanges, on peut penser que l'image constitue peut-être une modalité plus directe que le texte grâce à sa sémiotique non-linéaire, globale et non segmentée, contrairement au texte. Bien sûr, un tour composé d'une image (avec ou sans texte incrusté) peut aussi tout à fait avoir pour paire adjacente un tour plus classique constitué d'une phrase, ce qui est le cas dans cet autre échange : 


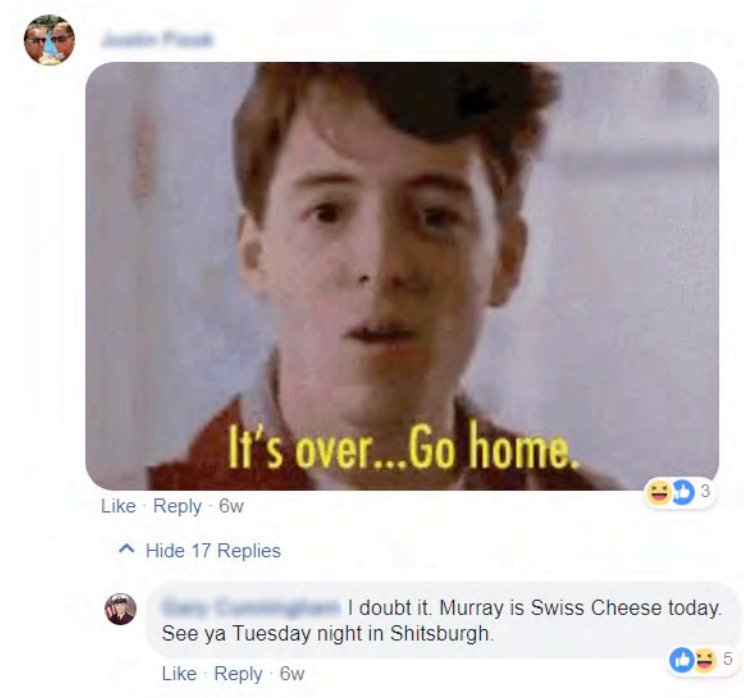

\section{Conclusion}

L'utilisateur de Facebook a désormais à sa disposition de nombreuses modalités pour s'exprimer. Celles-ci peuvent être verbales (le texte et son matériau lexical et morpho-syntaxique) ou non-verbales (émojis, émoticônes), mais aussi faire appel aux deux registres simultanément en intégrant potentiellement texte et image (gifs, autocollants, images, vidéos). La première conclusion qui s'impose au terme de cette étude est donc qu'il semble difficile de réduire les modalités iconiques à des composantes non-verbales de la communication. La deuxième conclusion concerne le rapprochement entre l'interaction en ligne et l'interaction face-à-face. En effet, plutôt qu'une révolution, il faut peut-être voir dans l'utilisation grandissante de modalités iconiques dans l'interaction sur Internet un mouvement d'enrichissement du discours médié par ordinateur qui la rapproche de l'interaction face-à-face. Cette dernière est "multicanale et pluricodique " (Kerbrat-Orecchioni 1998, 47) et fait appel à un matériau sémiotique foncièrement multimodal et non limité au verbal, comme les études gestuelles modernes (dont on a cité les ouvrages de référence plus haut) l'ont largement montré. L'enrichissement des modalités de l'interaction en ligne tend alors sans doute à la rapprocher du fonctionnement lui aussi multimodal de l'interaction orale en coprésence. 


\section{BIBLIOGRAPHIE}

ANDROUTSOPOULOS, Jannis. 2006. "Introduction : sociolinguistics and computer-mediated communication". Journal of Sociolinguistics 10(4) : 419-438.

ANDROUTSOPOULOS, Jannis \& HERRING, Susan C. 2015. "Computer-mediated discourse 2.0 ». In D. TANNEN, H.E. HAMILTON \& D. SCHIFFRIN (eds), The Handbook of Discourse Analysis. Hoboken : John Wiley, p. 127-151.

BIESWANGER, Markus \& INTEMANN, Frauke. 2011. "Patterns and variations in the language used in English-based discussion forums ». In M. LUGINBÜHHL \& D. PERRIN (eds), Muster und Variation: Median-linguistische Perspektiven auf Textproduktion und Text. Bern : Peter Lang, p. 157-187.

BIESWANGER, Markus. 2013. "Micro-linguistic structural features of computer-mediated communication ". In S.C. HERRING, D. STEIN \& T. VIRTANEN (eds), Pragmatics of computer-mediated Communication. Berlin : De Gruyter, p. 463-485.

CALBRIS, Geneviève \& PORCHER, Louis. 1989. Geste et communication. Paris : CREDIF-Hatier.

CALBRIS, Geneviève. 2011. Elements of Meaning in Gesture. Amsterdam : John Benjamins.

DRESNER, Eli \& HERRING, Susan C. 2010 : «Functions of the nonverbal in CMC: Emoticons and illocutionary force". Communication Theory $20: 249-268$.

DUCROT, Oswald \& SCHAEFFER, Jean-Marie. 1995. Nouveau dictionnaire encyclopédique des sciences du langage. Paris : Seuil.

HERRING, Susan C. 1996. Computer-Mediated Communication. Amsterdam : John Benjamins.

HERRING, Susan C. 2007. "A faceted classification scheme for computer-mediated discourse». Language@Internet, volume 4, issue 1 .

http://www.languageatinternet.org/articles/2007/761, consulté le 17 février 2018. 
HERRING, Susan C. 2012. "Grammar and Electronic Communication ". In C. CHAPELLE (ed.), Encyclopedia of applied linguistics. Hoboken : Wiley-Blackwell.

http://ella.slis.indiana.edu/ herring/e-grammar.2011.pdf, consulté le 17 février 2018.

HERRING, Susan C. 2013. "Discourse in Web 2.0 : familiar, reconfigured, emergent ". In D. TANNEN \& A.M. TRESTER (eds), Discourse 2.0 : language and the new media. Washington D.C. : Georgetown University Press, p. 1-26.

HERRING, Susan C. 2016. "New frontiers in multimodal communication ». In A. GEORGAKOPOULOU, T. SPILIOTI (eds), The Routledge Handbook of Language and Digital Communication. London : Routledge, p. 398-402.

HERRING, Susan C. \& DAINAS, Ashley. 2017. "Nice picture comment!': Graphicons in Facebook comment threads ». In Proceedings of the Fiftieth Hawaii International Conference on System Sciences. Los Alamitos, CA : IEEE.

https://scholarspace.manoa.hawaii.edu/bitstream/10125/41419/1/paper027 0.pdf, consulté le 6 mai 2018.

KENDON, Adam. 2004. Gesture: Visible Action as Utterance. Cambridge : Cambridge University Press.

KENDON, Adam. 1988. "How gestures can become like words ». In F. POYATOS (ed.), Cross-Cultural Perspectives in Nonverbal Communication. Toronto : Hogrefe, p. 131-141.

KERBRAT-ORECCHIONI, Catherine. 1998 [1990]. Les interactions verbales, tome I, troisième édition. Paris : Armand Colin.

McNEILL, David. 1992. Hand and Mind. Chicago : University of Chicago Press.

MILTNER, Kate \& HIGHFIELD, Tim. 2017. "Never gonna gif you up : analyzing the cultural significance of the animated GIF ». Social Media + Society, July-September $2017: 1-11$.

SCHNEEBELI, Célia. 2018. "Coding Emotion in Computer-Mediated Communication : The Example of YouTube Comments ». RANAM 51: 45-56. 
LES MODALITÉS ICONIQUES DANS LE DISCOURS MÉDIÉ

PAR ORDINATEUR : DU NEUF DANS L'INTERACTION ?

TOLINS, Jackson \& SAMERMIT, Patrawat. 2016. "GIFs as embodied enactments in text-mediated conversation ". Research on Language and Social Interaction 49(2): 75-91.

TRAVERSO, Véronique. 1999. L'analyse des conversations. Paris : Armand Colin. 